\title{
Significance of the Tryptophan Residue at the Low Affinity Saccharide-binding Site of Ricin $\mathbf{E}^{\dagger}$
}

\author{
Nobuyuki Yamasaki, Tomomitsu Hatakeyama, Hideki Ohba \\ and Gunki FunATSU \\ Laboratory of Biochemistry, Faculty of Agriculture, Kyushu University, \\ Hakozaki, Higashi-ku, Fukuoka 812, Japan
}

Received March 13, 1989

\begin{abstract}
Chemical modification of tryptophan residues in ricin $\mathbf{E}$ was investigated with regard to saccharide-binding. Two out of ten tryptophan residues in ricin $\mathrm{E}$ were modified with $\mathrm{N}$ bromosuccinimide at $\mathrm{pH} 4.5$ in the absence of specific saccharide accompanied by a marked decrease in the cytoagglutinating activity. Such a loss of the cytoagglutinating activity was found to be principally due to the oxidation of one tryptophan residue per B-chain. In the presence of lactose, one tryptophan residue/mol was protected from the modification with retention of a fairly high cytoagglutinating activity. However, GalNAc did not show such a protective effect. The binding of lactose to ricin $\mathrm{E}$ altered the environment of the tryptophan residue at the low affinity binding site of ricin $\mathrm{E}$, leading to a blue shift of the fluorescence spectrum and an UV-difference spectrum with a maximum at $290 \mathrm{~nm}$ and a trough at $300 \mathrm{~nm}$. The ability to generate such spectroscopic changes induced by lactose was retained in the derivative in which one tryptophan residue/mol was oxidized in the presence of lactose, but not in the derivative in which two tryptophan residues/mol were oxidized in the absence of lactose. Based on these results, it is suggested that one of the two surface-localized tryptophan residues is responsible for saccharide binding at the low affinity binding site of ricin $\mathrm{E}$, which can bind lactose but lacks the ability to bind GaINAc.
\end{abstract}

Ricin $\mathrm{E}$ is a toxic protein found in the smallgrain seeds of castor beans (Ricinus communis) grown in Japan. ${ }^{1)}$ This is a lactin with a $63,000 \mathrm{~mol}$ weight and consists of two nonidentical polypeptide chains (A- and B-chains) linked through a single disulfide bond. The Achain is responsible for inhibition of protein synthesis in eucaryotic cells and the B-chain serves to combine the toxin to the carbohydrate moiety on the cell membrane, ${ }^{2)}$ like ricin $D$ isolated from the large-grain seeds of Ricinus communis. ${ }^{3,4)}$ The molecular weight, toxicity for mice, and cytoagglutinating activity of ricin $\mathrm{E}$ is equal to those of ricin $\mathrm{D}$, but the two ricins differ in toxicity for cultured cells and affinities to Sepharose $4 \mathrm{~B}$ and Guar gum. ${ }^{2,5)}$ Such differences in the properties as are found between ricin $\mathrm{E}$ and ricin $\mathrm{D}$ have been considered to be attributable to the difference in the amino acid sequences of their Bchains. ${ }^{6)}$ Physicochemical studies on the binding of saccharides to ricin $E$ have suggested that the B-chain of ricin $E$ carries two saccharide-binding sites with different affinities, a high affinity binding site (HA-site) and a low affinity binding site (LA-site). ${ }^{7)}$ However, the nature of the saccharide-binding sites has not been elucidated completely. To shed further light on the structure-function relationship of ricin $\mathrm{E}$, tryptophan residues in this toxin were modified with $N$-bromosuccinimide and the properties of the resulting derivatives were examined. This paper describes the significance of the tryptophan residue at the

₹ This work was supported in part by a Grant-in-Aid for Scientific Research from the Ministry of Education, Science and Culture of Japan.

Abbreviations: $\quad$ NBS, $N$-bromosuccinimide; GalNAc, $N$-acetyl-D-galactosamine; HA-site, high affinity binding site; LA-site, low affinity binding site; UV, ultraviolet. 
LA-site of ricin E.

\section{Materials and Methods}

Ricin E was prepared by the method of Mise et al. ${ }^{1)}$ from small-grain castor bean seeds (Ricinus communis) cultivated in a field of Kyushu University. $N$-Bromosuccinimide and $N$-acetyl-D-galactosamine were obtained from Nacalai Tesque (Kyoto), and the former was recrystallized from distilled were before use. Lactose from Wako Pure Chemicals Industries Ltd. (Osaka) was used without further purification. Other reagents used in these experiments were analytical grade.

Protein concentration. The concentration of ricin $\mathrm{E}$ was measured spectrophotometrically using an absorption coefficient of $A_{1}^{1 \%} \mathrm{~cm}=15$ at $280 \mathrm{~nm} .{ }^{1 /}$ The concentration of the modified ricin $\mathrm{E}$ was measured by the method of Lowry et al. ${ }^{8)}$ using ricin $\mathrm{E}$ as a standard protein.

Oxidation with NBS. The oxidation of tryptophan residues with NBS was done essentially by the method of Spande and Witkop. ${ }^{9)}$ A $3 \mathrm{ml}$ sample of ricin $\mathrm{E}$ solution $(4.4 \mu \mathrm{M})$ in $0.1 \mathrm{M}$ acetate buffer at $\mathrm{pH} 4.0$ was placed in a quartz cell with a stirring bar. With stirring, $10 \mu \mathrm{l}$ of NBS solution at a given concentration in the same buffer was added, and after $5 \mathrm{~min}$ the absorption spectrum was recorded on a Hitachi 200-10 spectrophotometer. A control was prepared by addition of $10 \mu \mathrm{l}$ of the buffer solution in place of the NBS solution. To make the titration curve for NBS-oxidation of tryptophan in ricin $\mathrm{E}$, the NBS solution ( $2.8 \mathrm{~mm}$ ) was successively added until the decrease in the absorbance at $280 \mathrm{~nm}$ ceased. All reactions were done at $25^{\circ} \mathrm{C}$, and the number of tryptophan residues oxidized was calculated by the method described in the literature. $^{9 \text { ) }}$

Cytoagglutinating activity. Cytoagglutinating activities of ricin $\mathrm{E}$ and its derivatives were measured microscopically using Sarcoma 180 ascites tumor cells in $5 \mathrm{~mm}$ phosphate-buffered saline at $\mathrm{pH} \mathrm{7.1,} \mathrm{as} \mathrm{described} \mathrm{by}$ Funatsu et al. ${ }^{10)}$

Fluorescence spectra. Fluorescence spectra of ricin E and its derivatives were recorded on a Hitachi $650-10 \mathrm{~S}$ fluorescence spectrophotometer at $\mathrm{pH} 6.9$ and $25^{\circ} \mathrm{C}$ with excitation at $290 \mathrm{~nm}$. For measurements of the fluorescence spectra in the presence of lactose, $10 \mu \mathrm{l}$ of the mixture of protein and lactose was added to the protein solution. This was done in such a manner as to keep the protein concentration the same.

$U V$-difference spectra. UV-difference spectra of ricin $\mathrm{E}$ and its derivatives induced by binding with lactose were measured with a Hitachi 200-10 spectrophotometer at $\mathrm{pH}$ 6.9 and $25^{\circ} \mathrm{C}$. The spectra were measured by placing the mixture of protein and lactose in the sample cell and the same concentration of protein in the reference cell, by the method described previously. ${ }^{71}$

Isolation of the constituent polypeptide chains. Ricin E and modified ricin $\mathrm{E}$ were separated into their constituent polypeptide chains, essentially by the method of Olsnes and Pihl. ${ }^{1)}$ Proteins were treated with $2 \% 2$-mercaptoethanol in $10 \mathrm{~mm}$ Tris- $\mathrm{HCl}$ buffer at $\mathrm{pH} 8.5$ containing $0.1 \mathrm{M}$ lactose for $2 \mathrm{hr}$, and put on a column of DEAEcellulose $(1.5 \times 15 \mathrm{~cm})$ equilibrated with $10 \mathrm{~mm}$ Tris $-\mathrm{HCl}$ buffer at $\mathrm{pH} 8.5$. After being washed with the buffer used for equilibration, the column was first developed with $0.5 \mathrm{M} \mathrm{NaCl}$ in the same buffer and then with $0.1 \mathrm{M} \mathrm{NaOH}$. The unadsorbed fractions were pooled as the A-chain, and the fractions eluted with $0.5 \mathrm{M} \mathrm{NaCl}$ were collected as the $\mathrm{B}$-chain of ricin $\mathrm{E}$. In the case of the modified ricin $\mathrm{E}$, however, the B-chain was not recovered completely in the fractions eluted with $0.5 \mathrm{M} \mathrm{NaCl}$ and was eluted with $0.1 \mathrm{M}$ $\mathrm{NaOH}$. The fractions eluted with $0.1 \mathrm{M} \mathrm{NaOH}$ were immediately neutralized with $50 \%$ acetic acid, combined with the fractions eluted with $0.5 \mathrm{M} \mathrm{NaCl}$, and used as the $\mathrm{B}$ chain of the modified ricin E. Each of the fractions was dialyzed against deionized water, and analyzed spectroscopically.

\section{Results}

\section{NBS-oxidation of ricin $E$}

As demonstrated in Fig. 1, the absorption

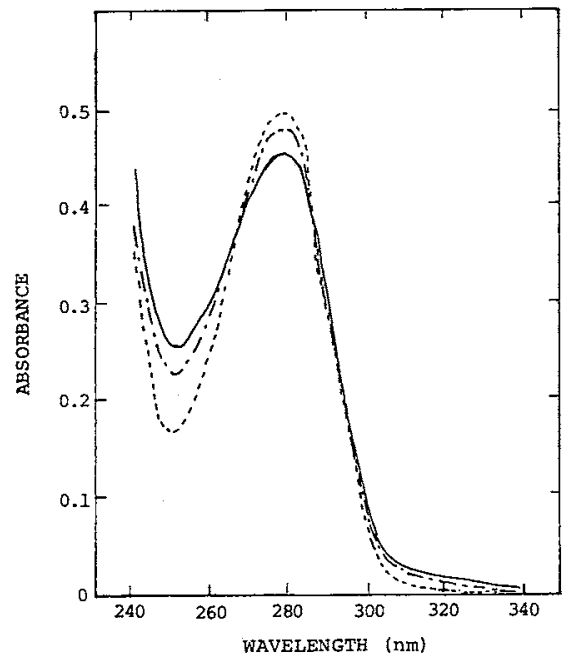

Fig. 1. Change in the Absorption Spectrum of Ricin E during NBS Oxidation.

To $3 \mathrm{ml}$ of ricin E solution $(4.4 \mu \mathrm{M})$ in $0.1 \mathrm{M}$ acetate buffer (pH 4.0) was added $10 \mu \mathrm{l}$ of a NBS solution in the same buffer. The spectra were recorded after $5 \mathrm{~min}$ of the addition of $10 \mu \mathrm{l}$ of the buffer solution (-----), $6 \mathrm{mM}$ NBS $(--)$ and $15 \mathrm{~mm}$ NBS (-) at $25^{\circ} \mathrm{C}$. 


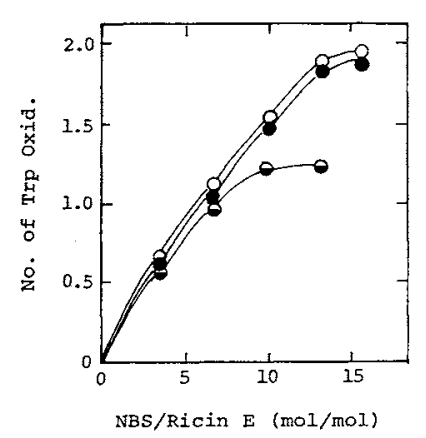

Fig. 2. Titration Curves for NBS Oxidation of Tryptophan in Ricin E and Its Complexes with Saccharides.

The oxidation reaction was done at $\mathrm{pH} 4.0$ and $25^{\circ} \mathrm{C}$ in the absence of saccharide $(O)$ and in the presence of $0.1 \mathrm{~mm}$ GalNAc (๑) or lactose $(\ominus)$. Other conditions were described under Materials and Methods.

Table I. CytoAgGlutinating Activities of Ricin E AND Its Derivatives

Ricin E was modified with NBS at $\mathrm{pH} 4.5$ and $25^{\circ} \mathrm{C}$. Details are described under Materials and Methods.

\begin{tabular}{lcc}
\hline Protein & $\begin{array}{c}\text { Moles of Trp } \\
\text { oxidized per } \\
\text { mole of ricin E }\end{array}$ & $\begin{array}{c}\text { Cytoagglutina- } \\
\text { ing activity } \\
(\%)^{a}\end{array}$ \\
\hline $\begin{array}{l}\text { Native ricin E } \\
\text { Ricin E modified in the } \\
\text { absence of lactose }\end{array}$ & 0 & 100 \\
$\begin{array}{l}\text { Ricin E modified in the } \\
\text { presence of } 0.1 \mathrm{M} \text { lactose }\end{array}$ & $1.9^{b}$ & 3 \\
\hline
\end{tabular}

a Measured using Sarcoma 180 ascites tumor cells at pH 7.1. ${ }^{10}$ For cytoagglutination tests, samples of native and oxidized ricin $\mathrm{E}$ were dialyzed against $5 \mathrm{~mm}$ phosphate buffered saline $(\mathrm{pH} 7.1)$ at $4^{\circ} \mathrm{C}$.

$b$ Number of residues eventually modified under the conditions cited.

spectrum of ricin E changed greatly on NBSoxidation. However, the absorbance at $265 \mathrm{~nm}$ remained unchanged during NBS-oxidation and an isosbestic point was found at this wavelength. The titration curves for NBSoxidation of tryptophan residues in ricin $\mathrm{E}$ in the absence and presence of saccharide are shown in Fig. 2. The extent of tryptophan modification increased with increasing the amount of NBS added, and 1.9 tryptophan residues/mol were eventually oxidized with a 16-fold excess of NBS at $\mathrm{pH} 4.0$ in the absence of lactose. In the presence of $0.1 \mathrm{~m}$ lactose, however, only 1.2 tryptophan residues $/ \mathrm{mol}$ were modified, and 0.7 tryptophan residues/ mol were protected from this modification reaction. Such a protective effect of saccharide on this modification reaction was not observed when GalNAc was used in place of lactose. Table I shows the effects of tryptophan modification on the cytoagglutinating activity of ricin E. The oxidation of tryptophan with NBS led to a marked decrease in the cytoagglutinating activity of ricin $E$ toward Sarcoma 180 ascites tumor cells, and only $3 \%$ of the residual activity was found in the derivative, 1 -oxa-ricin $\mathrm{E}$, in which 0.8 tryptophan residues/mol were converted to oxindolealanine in the absence of lactose. The cytoagglutinating activity of another derivative, 2oxa-ricin E, in which 1.9 tryptophan residues/ mol were oxidized, was less than $1 \%$ that of the native ricin $E$. On the contrary, when the modification reaction was done in the presence of $0.1 \mathrm{~m}$ lactose, the resulting derivative, 1-oxa-ricin E(lac), which contained 1.2 oxindolealanine/mol, retained fairly high cytoagglutinating activity $(33 \%)$. These results showed a fairly good agreement with those obtained for the NBS-oxidation of tryptophan in ricin $\mathrm{D}$ isolated from the large-grain castor bean seeds. ${ }^{12)}$

\section{Fluorescence spectra of native and modified ricin $E$}

Figure 3 shows the fluorescence spectra of oxidized ricin $E$ in the absence and presence of lactose compared with those of native ricin $\mathrm{E}$. Upon excitation at $290 \mathrm{~nm}$, ricin $\mathrm{E}$ had a fluorescence emission spectrum with a maximum at $335 \mathrm{~nm}$. The fluorescence intensity decreased greatly upon the oxidation of tryptophan with NBS with a shift of the emission maximum to a shorter wavelength. The fluorescence intensity of the inactive derivative, 2-oxa-ricin E, was only $43 \%$ that of native ricin $E$. The extent of the loss of the fluorescence of ricin $\mathrm{E}$ by the present modification reaction was almost identical to that found in the NBS-oxidation of tryptophan in 


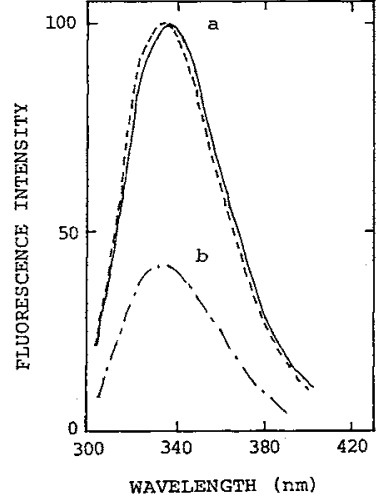

Fig. 3. Fluorescence spectra of Ricin E and 2-Oxa-ricin $\mathrm{E}$ in the Absence and Presence of Lactose.

Fluorescence spectra were recorded in $4.4 \mathrm{~mm}$ phosphate buffer ( $\mathrm{pH} \mathrm{6.9)}$ with $\mathrm{NaCl}$ added to give 0.1 ionic strength at $25^{\circ} \mathrm{C}$. Protein concentration was $0.57 \mu \mathrm{M}$ and the excitation wavelength was $290 \mathrm{~nm}$. a, fluorescence spectra of ricin $E$ in the absence $(-$ ) and presence of $0.1 \mathrm{~mm}$ lactose (-----); b, fluorescence spectrum of 2-oxa-ricin $E$ in the absence or presence of $0.1 \mathrm{~mm}$ lactose.

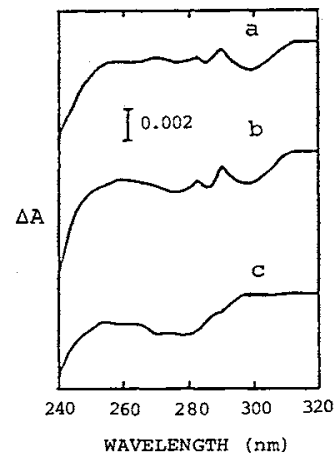

Fig. 4. Difference Spectra of Ricin E and Its Derivatives Induced by Binding with Lactose.

The UV-difference spectra were recorded by placing the mixture of protein and lactose in the sample cell and the same concentration of protein in the reference cell, by the method described previously. ${ }^{7}$ Measurements were done in the same buffer as described in Fig. 3 and at $25^{\circ} \mathrm{C}$. The final concentrations of protein and lactose were $0.1 \mathrm{~mm}$ and $11 \mu \mathrm{M}$, respectively. a, ricin $\mathrm{E}$; b, 1-oxa-ricin $\mathrm{E}(\mathrm{lac}) ; \mathrm{c}, 2$-oxa-ricin E.

ricin $\mathrm{D}(45 \%) .{ }^{12)}$ Upon addition of lactose, the emission maximum of ricin $E$ shifted from $335 \mathrm{~nm}$ to $332 \mathrm{~nm}$. Such a change in the fluorescence spectrum, however, was not observed when lactose was added to 2-oxa-ricin E.

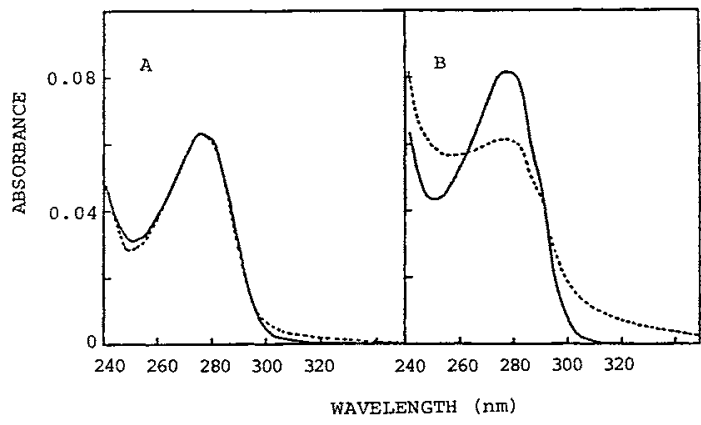

Fig. 5. UV-Absorption spectra of the Constituent Polypeptide Chains of Native and Modified Ricin E.

The spectra of the constituent polypeptide chain $(1.6 \mu \mathrm{M})$ were measured in $5 \mathrm{~mm}$ phosphate buffer ( $\mathrm{pH} 7.0$ ) containing $0.14 \mathrm{~mm} \mathrm{NaCl}$. Panels $\mathrm{A}$ and $\mathrm{B}$ shows the spectra of the A-chains and the B-chains, respectively.

\section{$U V$-difference spectra of native and modified ricin $E$ induced by lactose binding}

The lactose-induced UV-difference spectra of ricin $\mathrm{E}$ and its derivatives are demonstrated in Fig. 4. As previously reported, the UVdifference spectrum of the ricin E-lactose complex versus ricin $E$ had a trough at $300 \mathrm{~nm}$ and a small but distinct positive peak at $290 \mathrm{~nm}$. An almost identical difference spectrum was obtained for the active derivative, 1-oxa-ricin $\mathrm{E}(\mathrm{lac})$. However, the UV-difference spectrum generated by the inactive derivative, 2-oxaricin $\mathrm{E}$, was different in shape from that given by native ricin $E$, was different in shape from that given by native ricin $E$, and neither the trough at $300 \mathrm{~nm}$ nor the positive peak at $290 \mathrm{~nm}$ was found in the difference spectrum.

\section{$U V$-absorption spectra of the $A$-and $B$-chains of the modified ricin $E$}

In Fig. 5, the UV-absorption spectra of the constituent polypeptide chains isolated from 2oxa-ricin $\mathrm{E}$ are compared with those obtained from native ricin $E$. It is evident that the absorption spectra of the A-chains of ricin $\mathrm{E}$ and its derivative were almost the same. On the other hand, the shape of the absorption spectrum of the B-chain of 2-oxa-ricin E was quite different from that of native ricin $E$. The absorption spectrum of the B-chain of the modified ricin $\mathrm{E}$ was characterized by a de- 
crease in the intensity at $280 \mathrm{~nm}$ and an increase in the intensity at $250 \mathrm{~nm}$. These results indicate that in 2-oxa-ricin $\mathrm{E}$ the modified tryptophan residues are contained in the Bchain but not in the A-chain.

\section{Discussion}

The reactivity of tryptophan toward NBS has been used as a criterion for discrimination of the states of tryptophan residues in proteins. ${ }^{9)}$ Ricin E contains ten tryptophans per molecule: one in the A-chain and the others in the B-chain. ${ }^{6}$ As demonstrated in this study, two out of ten tryptophan residues in ricin $E$ are eventually modified with NBS in the $a b-$ sence of lactose (Fig. 2). The UV-absorption spectra of the constituent polypeptide chains isolated from the modified ricin E clearly indicate that the oxidized tryptophan residues are contained only in the B-chain (Fig. 5). These findings suggest that two of the nine tryptophan residues in the B-chain are at or near the surface of the ricin $E$ molecule with a fairly high susceptibility to NBS oxidation. Such a view can be supported by the fluorescence spectroscopic data. The fluorescence emission maximum at $335 \mathrm{~nm}$ as found in the fluorescence spectrum of ricin $\mathrm{E}$ is characteristic of tryptophan and solely due to the exposed tryptophan. ${ }^{13,14)}$ Oxidation of two tryptophan residues $/ \mathrm{mol}$ caused a $57 \%$ loss of the fluorescence of ricin E (Fig. 3). This implies that most of the fluorescence of ricin $E$ is contributed by the two tryptophans susceptible to the NBS oxidation.

It is noteworthy that the cytoagglutinating activity of ricin $\mathrm{E}$ decreased greatly during NBS oxidation, and such a loss of the cytoagglutinating activity is principally due to the modification of one tryptophan residue $/ \mathrm{mol}$ (Table I). In the presence of lactose, however, one tryptophan residue/mol escaped from this modification reaction with a retention of the cytoagglutinating activity, suggesting the involvement of one tryptophan residue in the saccharide binding of ricin $\mathrm{E}$. There is a possibility that NBS attacks both tryptophan and tyrosine in proteins. However, the modification of tyrosine in this case can be disregarded by the evidence that the isosbestic point at $263 \mathrm{~nm}$ in the UV-absorption spectrum was retained during the NBS-oxidation. If tyrosine residues are modified with NBS, such a isosbestic point should not be obtained. ${ }^{15)}$ Accordingly, the lower cytoagglutinating activity of the active derivative, 1-oxa-ricin $\mathrm{E}$ (lac), compared to native ricin $\mathrm{E}$ may be explained by some conformational changes induced by modification of an additional tryptophan residue other than the tryptophan at the LA-site.

Physicochemical studies on the interaction of ricin $\mathrm{E}$ with its specific saccharides have indicated that the B-chain carries two saccharide-binding sites with different affinities, the high affinity binding site (HA-site) and the low affinity binding site (LA-site). ${ }^{7}$ As demostrated here, the binding of lactose to ricin $\mathrm{E}$ induces spectroscopic changes in this lectin (Figs. 3 and 4). A trough at $300 \mathrm{~nm}$ in the lactose-induced UV-difference spectrum has been interpreted as being due to a change in the environment of tryptophan ${ }^{16)}$ and is representative of the specific binding of lactose to the LA-site of ricin E, as well as the blue shift of the fluorescence spectrum induced by lactose binding. ${ }^{6)}$ Such a trough, however, is not found in the UV-difference spectrum generated by 2-oxa-ricin E (Fig. 4), and there is no change in the fluorescence spectrum of this derivative upon the addition of lactose (Fig. 3). These observation suggest the lack of the binding ability at the LA-site in the inactive derivative, 2-oxa-ricin E. On the contrary, the active derivative, 1-oxa-ricin E(lac) has a lactose-induced UV-difference spectrum identical to that given by native ricin $E$. This supports the above view and suggests that in 1oxa-ricin $\mathrm{E}(\mathrm{lac})$ the tryptophan residue at the LA-site remains unmodified and the binding ability at this site is retained. On the other hand, GalNAc does not have any effect on the NBS-oxidation of tryptophan in ricin $\mathrm{E}$ (Fig. 2). Spectroscopic studies on the binding of saccharide to ricin $\mathrm{E}$ have suggested that the 
HA-site can bind both galactopyranoside and GalNAc, while the LA-site can bind galactopyranosides but lacks the ability to bind GalNAc. ") Taking these results into account, we postulate that lactose binds to ricin $\mathrm{E}$ in such a manner as to prevent the access of NBS to the tryptophan residue at the LA-site, but GalNAc is bound only by the HA-site without any effect on the susceptibility of the tryptophan residue at the LA-site to NBS.

The results mentioned above provide further insight into the saccharide-binding site of ricin E. It should be noticed that the susceptibilities of tryptophan residues to NBS in ricin E (Fig 2) are almost identical to those in ricin $D,{ }^{12)}$ in spite of the difference in amino acid sequence of the B-chain between two ricins. ${ }^{7)}$ As well as in the case of ricin $E$, ricin $D$ found in the large-grain castor bean seeds has two saccharide-binding sites (HA- and LA-sites) per B-chain which is composed of two domains, a $\mathrm{N}$-terminal half domain and a $\mathrm{C}$ terminal half domain: the HA-site containing $\mathrm{Tyr}_{248}$ is on the C-terminal half domain and the LA-site containing $\operatorname{Trp}_{37}$ on the $\mathrm{N}$ terminal half domain. ${ }^{19 \sim 21)} \mathrm{A}$ comparison of the amino acid sequence of the B-chain of ricin $E$ with those of other castor bean lectins have revealed that the B-chain of ricin E is composed of the $\mathrm{N}$-terminal half of ricin $\mathrm{D}$ and the $\mathrm{C}$-terminal half of castor bean hemagglutinin, $\left.{ }^{6}\right)$ another type of lectin which is relatively non-toxic but a hemagglutinating protein. ${ }^{4)}$ In a preceding paper, we suggested that the saccharide-binding ability of the HA-site of ricin $\mathrm{E}$ was lower than that of ricin $\mathrm{D}$, but there was no appreciable difference in the binding ability at the LA-site between two ricins. ${ }^{77}$ Assuming that the B-chain of ricin E has a domain structure similar to that of ricin $\mathrm{D}$, it can be deduced that the LA-site of ricin $E$ has the same conformation as that of ricin $D$ and thereby the tryptophan residue which is important for saccharide binding at this site shows the same reactivity toward NBS as the tryptophan residue present at the LA-site of ricin D.

\section{References}

1) T. Mise, G. Funatsu, M. Ishiguro and M. Funatsu, Agric. Biol. Chem., 41, 2041 (1977).

2) G. Funatsu, T. Mise, H. Matsuda and M. Funatsu, Agric. Biol. Chem., 42, 851 (1978).

3) S. Benson, S. Olsnes, A. Pihl, J. Skorve and A. K. Abraham, Eur. J. Biochem., 53, 573 (1975).

4) G. L. Nicolson, J. Blaustein and M. E. Etzler, Biochemistry, 13, 196 (1974).

5) T. Hatakeyama, H. Ohba, N. Yamasaki and G. Funatsu, Agric. Biol. Chem., 53, (1989), in press.

6) T. Araki and G. Funatsu, Biochim. Biophys. Acta, 914, 191 (1987).

7) T. Hatakeyama, H. Ohba, N. Yamasaki and G. Funatsu, J. Biochem., 105, 444 (1989).

8) O. H. Lowry, N. J. Rosebrough, A. L. Farr and R. J. Randall, J. Biol. Chem., 193, 265 (1951).

9) T. F. Spande and B. Witkop, in "Method in Enzymology," Vol. 11, ed. by C. H. W. Hirs, Academic Press, New York, 1967, pp. 498 532.

10) G. Funatsu, S. Miyauchi and M. Funatsu, Agric. Biol. Chem., 40, 639 (1976).

11) S. Olsnes and A. Pihl, Biochemistry, 12, 312 I (1973).

12) T. Hatakeyama, N. Yamasaki and G. Funatsu, $J$. Biochem., 99, 1049 (1986).

13) R. C. Kelley and P. H. VonHippel, J. Biol. Chem., 252, 7299 (1976).

14) M. S. Herrmann and D. W. Behnke, Biochim Biophys. Acta, 629, 43 (1980).

15) S. R. Patanjali, M. J. Swamy, V. Anatharam, M. I. Khan and A. Sulolia, Biochem. J., 217, 773 (1984).

16) V. S. Anahtarayanan and C. C. Begelow, Biochemistry, 8, 3713 (1969). 